\title{
A Ghost in Coronary Artery - Coronary Artery Embolism After Discontinuation of Rivaroxaban in a Patient With Atrial Fibrillation: Case Report and Review of Literature
}

\author{
Danish Abbasi ${ }^{1}$, Negar Salehi ${ }^{2}$, Saif Faiek ${ }^{3}$, Waqas J. Siddiqui ${ }^{4,5}$, Shahzed Ahmad ${ }^{6}$ \\ 1. Cardiovascular Diseases, University of Arkansas, Little Rock, USA 2. Internal Medicine - Cardiology, University of \\ Arkansas, Little Rock, USA 3. Internal Medicine, AtlantiCare Regional Medical Center, Atlantic City, USA 4. \\ Cardiology/Nephrology, Drexel University College of Medicine, Philadelphia, USA 5. Cardiology/Nephrology, Orange \\ Park Medical Center, Orange Park, USA 6. Cardiovascular Disease, Lower Bucks Hospital, Bristol, USA
}

Corresponding author: Saif Faiek, saif.almomaiz@gmail.com

\begin{abstract}
Coronary artery embolism (CAE) is a rare clinical entity that can cause acute myocardial infarction (AMI). The exact prevalence of coronary artery embolism is unknown. CAE was found to be associated with conditions that can lead to thrombo-embolism, including infective endocarditis, atrial fibrillation, mitral valve disease, valve surgery. Herein, we report a 78-year-old male with a past medical history of atrial fibrillation on rivaroxaban who presented to the hospital emergency department complaining of chest pain. The patient's anticoagulation therapy was recently held due to a concern for gastrointestinal bleeding. After further evaluation of the patient's symptoms and reviewing his electrocardiogram (ECG) which showed STdepression in lateral leads and ST-elevation in aVR, urgent cardiac catheterization was done which showed left main coronary artery thrombosis extending into the left anterior descending artery (LAD) and left circumflex artery (LCX). The patient was started on a heparin drip and underwent a successful aspiration thrombectomy with subsequent improvement in his symptoms.
\end{abstract}

Received 08/13/2020 Review began 08/18/2020 Review ended 08/19/2020 Published 08/27/2020

๑) Copyright 2020 Abbasi et al. This is an open access article distributed under the terms of the Creative Commons Attribution License CC-BY 4.0., which permits unrestricted use, distribution, and reproduction in any medium, provided the original author and source are credited.
Categories: Cardiac/Thoracic/Vascular Surgery, Cardiology, Hematology

Keywords: coronary artery embolism, acute myocardial infarction, rivaroxaban, xarelto, anticoagulation

\section{Introduction}

Coronary artery embolism (CAE) is a rare cause of acute myocardial infarction (AMI) and should be suspected when AMI occurs in patients with conditions associated with embolism. It is associated with high mortality. Atrial fibrillation (AF) is an associated risk factor for CAE. Discontinuation of anticoagulation, especially rivaroxaban, is associated with a higher risk of embolism [1, 2]. In 1953 Cheng et al. reported 53 cases of CAE. Most of them were diagnosed on autopsy [3]. Before 1960, more than half of CAE were secondary to infective endocarditis [4-6]; however, recent data has shown that coronary embolism is mainly related to atrial fibrillation $[6,7]$.

\section{Case Presentation}

A 78-year-old male with a past medical history of hypertension, chronic kidney disease, chronic obstructive pulmonary disease, and atrial fibrillation with CHA2DS2-VASc score of 3 on rivaroxaban, presented to the hospital with complaints of left upper quadrant pain and chest pain. The patient was recently admitted with a concern for gastrointestinal bleeding, and rivaroxaban was held. Unfortunately, the patient left the hospital against medical advice at that time without further workup for gastrointestinal bleeding. The patient presented two days after leaving the hospital. On physical examination, the patient was alert and oriented. The abdomen was soft with mild left upper quadrant tenderness. Lungs were clear to auscultation without any crackles or wheezing. The patient had an irregular heart rhythm, and no murmurs were appreciated. The patient's vital signs were stable, except for his heart rate of $100 \mathrm{bpm}$. The patient underwent a stat computed tomography angiography of the chest, which was negative for aortic dissection and pulmonary embolism. Other laboratory workup showed hemoglobin $9.1 \mathrm{gm} / \mathrm{dl}$ (reference range $13-17 \mathrm{gm} / \mathrm{dl}$ ), hematocrit $27.5 \%$ (reference range 41-50\%), and lipase $170 \mathrm{U} / \mathrm{L}$ (reference range 0-160 U/L). The patient had an electrocardiogram (ECG), which showed atrial fibrillation with a ventricular rate of $112 \mathrm{bpm}$, right bundle branch block, ST-segment depression in lateral leads with ST-segment elevation in aVR (Figure 1). His Troponin level was minimally elevated at $0.036 \mathrm{ng} / \mathrm{ml}$ (normal range below $0.04 \mathrm{ng} / \mathrm{ml}$ ) with a brain natriuretic peptide (BNP) of $436 \mathrm{pg} / \mathrm{ml}$ (normal range below $100 \mathrm{pg} / \mathrm{ml}$ ). 


\section{Cureus}

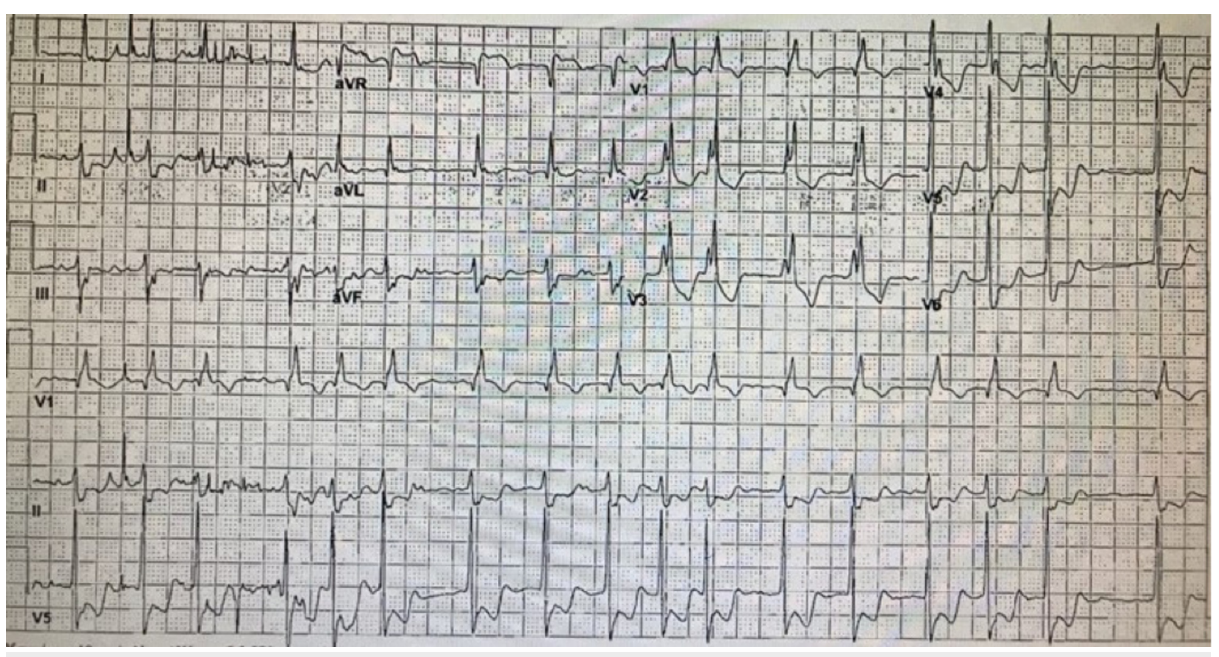

FIGURE 1: ECG - ST-elevation in aVR, depression in V5 and V6

The patient was transferred for urgent cardiac catheterization after heparin drip initiation, which showed left-main thrombosis with extension into the left anterior descending artery (LAD) with $90 \%$ obstruction and left circumflex artery (LCX) leading to $100 \%$ occlusion without collaterals (Figure 2A, 2B). The mid to distal right coronary artery (RCA) had minimal luminal irregularities. After insertion of the intra-aortic balloon pump (IABP) for hemodynamic support, the left main was engaged, and two 0.014 wires were crossed across the LAD and LCX lesions. Aspiration thrombectomy of LAD was performed that caused the distal embolization of the thrombus (Figure 2C). Repeat thrombectomy was done. Post-procedure there was TIMI grade 3 flow throughout the LCX (Figure 2D) without any evidence of thrombosis, dissection, or distal embolization. The patient transferred to the critical care unit. The heparin infusion and abciximab were continued, repeated ECG showed complete resolution of ST-segment elevation in aVR and depression in anterolateral leads (Figure 3). The patient was subsequently taken off IABP support. The patient was stable without any chest pain and was discharged on rivaroxaban. 


\section{Cureus}

A

B
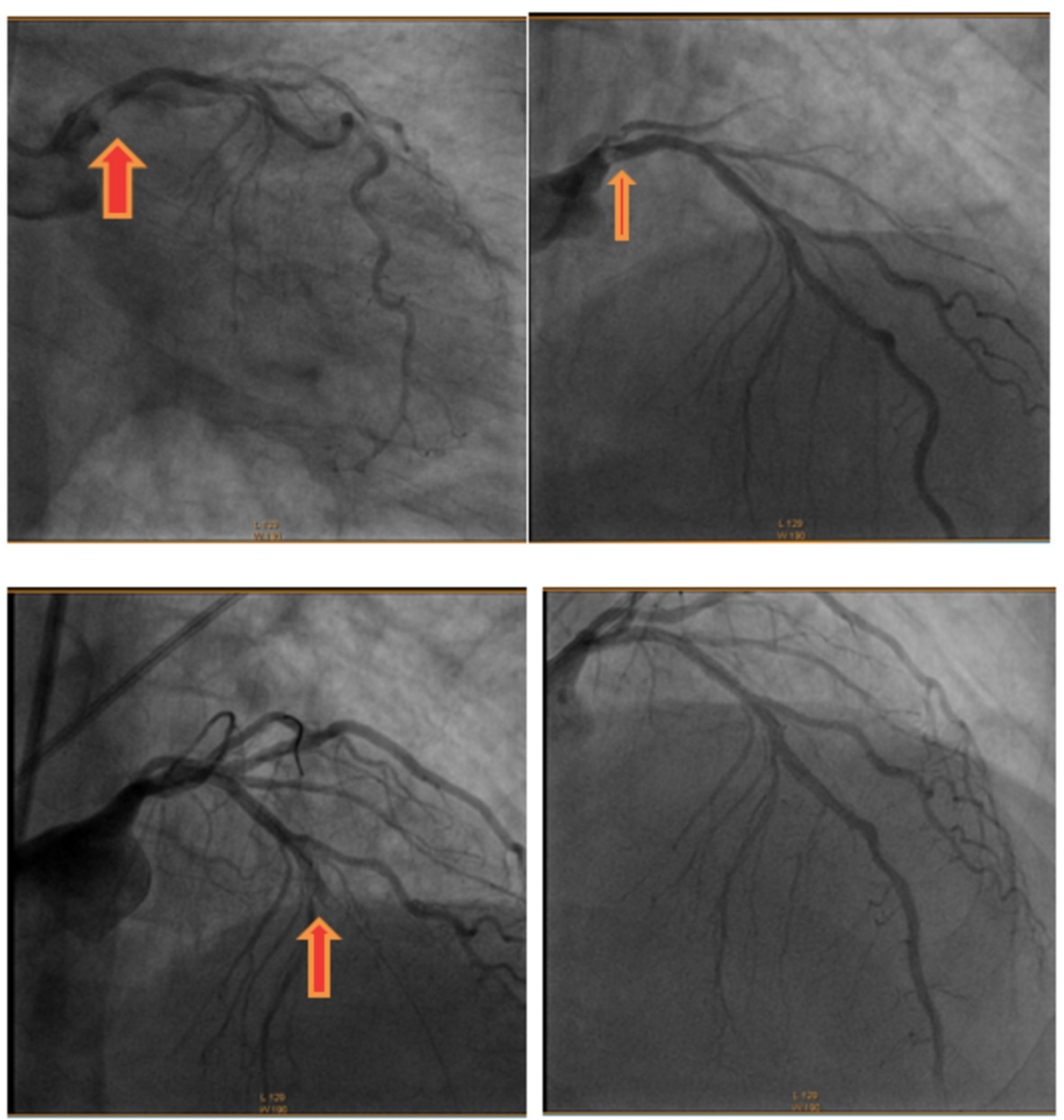

$\mathrm{C}$

D

FIGURE 2: (A) Left coronary angiogram showing left- main thrombus.

(B) Left angiogram showing left main, circumflex and ostial LAD thrombus. (C) Left coronary angiogram showing mid LAD occlusion due to distal embolization of thrombus. Note restoration of TIMI grade 3 flow into LCX and clear left-main. (D) Left coronary angiogram showing clear left main and left circumflex artery with distal LAD thrombus.

LAD: Left anterior descending artery; LCX: Left circumflex artery. 


\section{Cureus}

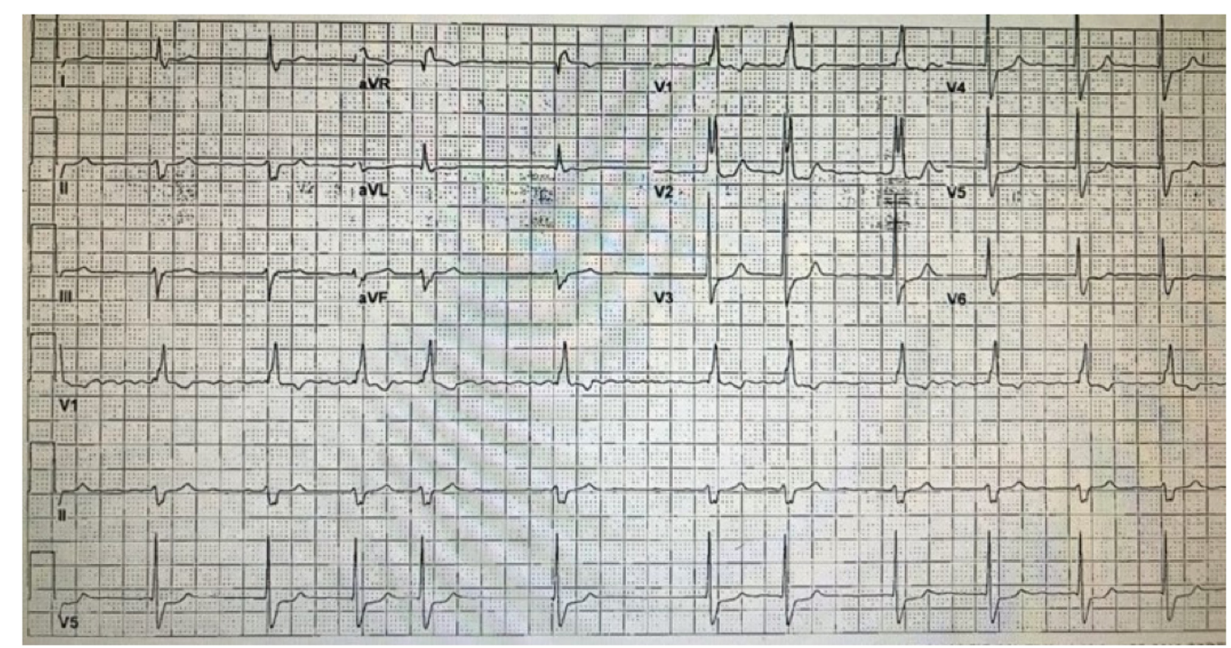

FIGURE 3: ECG - Resolution of ST-segment changes after intervention

\section{Discussion}

Coronary artery embolism is a rare cause of acute myocardial infarction and should be suspected in patients with conditions associated with thrombo-embolism. Virchow described the first case of this type in 1856 [1]. Saphir in 1933 published a review of 16 cases of coronary embolism, including a report of three individual cases [2]. By 1953 Cheng et al. reported 53 cases of CAE. Most of the cases diagnosed on autopsy; only three diagnosed during life [3]. Before 1960, infective endocarditis [4-6] accounted for more than half of all cases; however, the rate has declined since then [1]. Recent data has shown that coronary embolism is mainly related to atrial fibrillation $[6,7]$. There are other reports about CAE that included but are not limited to mitral valve disease [8], pulmonary resection [9] and prosthetic heart valve [10].

The exact prevalence of coronary artery embolism is unknown. An extensive review by Shibata et al. [11] of the National Cerebral and Cardiovascular Center (NCVC) AMI database noted that the prevalence of CAE was $2.9 \%(n=52)$, including eight $(15 \%)$ patients with multi-vessel CAE. The study reviewed 1,776 patients with de novo AMI and found that the most common cause was atrial fibrillation (73\%). Five patients of atrial fibrillation had a recurrence CAE with a median follow-up of 49 months, CAE, and thromboembolism recurred in five AF patients. Prizel et al. in 1978 analyzed autopsies of 55 patients with CAE [12]. The study found heart disease (40\%), cardiomyopathy (29\%), coronary atherosclerosis (16\%), and chronic atrial fibrillation (24\%) in those patients. Mural thrombi were present in 18 (33\%), with clinically diagnosed myocardial infarction in 15 (27\%) and death in 11 (20\%) patients. Most emboli involved the left coronary artery. The authors of the study concluded that coronary emboli might produce signs and symptoms identical to atherosclerotic coronary disease. Distal embolization was a common cause of infarcts that resulted in small transmural myocardial infarction. Kolodgie et al. noted that coronary arteries are relatively protected from emboli, in comparison with other organs [13]. Reasons for decreased reported incidence also include failure to distinguish embolism from thrombosis or an inability to do a systematic search for small emboli in the distal and intramural branches of coronary arteries. The two significant determinants are the size of the embolus and the size of the lumen of the artery that will be affected. Smaller emboli are more likely to go distal to a small coronary arterial segment and decrease the probability of MI or fatal arrhythmia [14].

Prompt identification and management are essential, as the higher incidence of all-cause death (hazard ratio, 3.82) and cardiac death (hazard ratio, 5.39) have been reported in patients with CAE as compared to non-CAE cohorts. Like in our patient, the DeWinter findings on the EKG of aVR elevation and ST-segment depression in precordial leads were suggestive of left main occlusion or proximal LAD stenosis. Patients with coronary artery embolism appear to be a high-risk sub-group of acute myocardial infarction individuals in the long term. Kaplan-Meier analysis of patients by Shibata et al. also showed similar findings (hazard ratio, 9.29) [11]. The unexpectedly higher mortality rates in the CAE group may be related to comorbidities or lack of collaterals in the native arteries that cause more extensive infarct and more muscle loss.

A coronary angiogram is a diagnostic procedure of choice for CAE. The NCVC proposed three major and three minor criteria for the clinical diagnosis of CAE, which is most commonly used [15]. Diagnosis of coronary embolism is classified as Definite (when two or more major criteria, or one major plus $\geqslant 2$ minor criteria, or three minor criteria are present) vs. Probable (when one major criterion plus one minor criterion, or two minor criteria are present) (Table 1). 


\section{Cureus}

Major criteria: 1- Angiographic evidence of coronary artery embolism and thrombosis without atherosclerotic components. 2Concomitant coronary artery embolization at multiple sites. 3- Concomitant systemic embolization without left ventricular thrombus attributable to acute myocardial infarction.

Minor criteria: 1- <25\% stenosis on coronary angiography, except for the culprit lesion. 2- Evidence of an embolic source based on transthoracic echocardiography, transesophageal echocardiography, computed tomography, or MRI (magnetic resonance imaging). 3- Presence of embolic risk factors: Atrial fibrillation, cardiomyopathy, rheumatic valve disease, prosthetic heart valve, patent foramen ovale, atrial septal defect, history of cardiac surgery, infective endocarditis, or hypercoagulable state.

Definite CAE (coronary artery embolism): two or more major criteria, or one major criterion plus $\geq 2$ minor criteria, or three minor criteria

Probable CAE: one major criterion plus one minor criterion, or two minor criteria.

A diagnosis of CE should not be made if there is: pathological evidence of atherosclerotic thrombus, history of coronary revascularization, coronary artery ectasia, plaque disruption or erosion detected by intravascular ultrasound or optic coherence tomography in the proximal part of the culprit lesion

TABLE 1: Proposed National Cerebral and Cardiovascular Center criteria for the clinical diagnosis of coronary artery embolism

Diagnosis of CAE should not be made if there is pathological evidence of atherosclerotic thrombus, history of coronary revascularization, or coronary artery ectasia.

This case had two major criteria and one minor criterion. The angiographic evidence of coronary artery embolism and concomitant embolization at multiple sites are major, and the presence of atrial fibrillation in the patient is a minor criterion. Based on the NCVC criteria, the patient was classified as Definite Coronary Embolism.

In literature, there is no consensus regarding optimal therapeutic algorithms for the management of embolic MI. In the review by Shibata et al., 58\% of patients underwent percutaneous coronary intervention (PCI), $97 \%$ of these patients had thrombus aspiration as the initial strategy [11]. Conservative management was observed in $42 \%$ of the patients with distal occlusion or small vessel involvement. Hernández et al. successfully treated three patients of coronary embolism with transluminal coronary angioplasty and stenting [16]. Pifarre et al. reported the use of catheter embolectomy combined with aortocoronary vein bypass graft in four patients [17].

Frikha et al. reviewed six patients diagnosed with coronary artery embolism [18]. Risk factors for coronary embolism include rheumatic mitral stenosis, mitral valve replacement, and atrial fibrillation. Three patients were managed with aspiration, while thrombolysis was performed in three cases. All patients had a favorable outcome.

Belli et al. recommended a strategy of combining maximal antiplatelet therapy, glycoprotein IIb/IIIa inhibitor infusion, direct aspiration of thrombus along with adjunctive mechanical protection with a balloon and catheter system to protect from distal embolization [19]. Successful aspiration of thrombus was obtained in seven out of eight attempted procedures, with an inability to negotiate the angulated take-off of the LCX artery in one patient. Small distal emboli not amenable to intervention can be managed with anticoagulation.

Rivaroxaban (Xarelto) is an oral once a day factor Xa inhibitor medication for the prevention of stroke and embolism in atrial fibrillation. The ROCKET AF study verified the safety of rivaroxaban as a substitute to warfarin for the prevention of thromboembolism in non-valvular AF [20]. Discontinuation of rivaroxaban has been attributed to a rebound phenomenon with a higher risk of stroke or thromboembolic phenomenon. Initial trial data showed a similar rate of complications between the drugs; however, the higher event rate was seen with rivaroxaban from three to 30 days after study drug discontinuation.

In our patient, the discontinuation of rivaroxaban increased the risk of embolism. The patient was managed with aspiration thrombectomy with percutaneous transluminal coronary angioplasty of the distal LAD, with the resolution of symptoms.

\section{Conclusions}

Coronary embolism is a rare but known cause of acute myocardial infarction with a higher incidence of allcause death. Abrupt discontinuation of anticoagulation, particularly rivaroxaban, should bring more attention toward clinical suspicion for coronary embolism in atrial fibrillation patients. This case report 


\section{Additional Information \\ Disclosures}

Human subjects: Consent was obtained by all participants in this study. Conflicts of interest: In compliance with the ICMJE uniform disclosure form, all authors declare the following: Payment/services info: All authors have declared that no financial support was received from any organization for the submitted work. Financial relationships: All authors have declared that they have no financial relationships at present or within the previous three years with any organizations that might have an interest in the submitted work. Other relationships: All authors have declared that there are no other relationships or activities that could appear to have influenced the submitted work.

\section{References}

1. Charles RG, Epstein EJ: Diagnosis of coronary embolism: a review . J R Soc Med. 1983, 76:863-869. 10.1177/014107688307601012

2. Shrader EL, Bawell MB, Mioragues V: Coronary embolism. Circulation. 1956, 14:1159-1163.

3. Cheng JTO, Cahill WJ, Foley EF: Coronary embolism. JAMA. 1953, 153:211-213. 10.1001/jama.1953.02940200033008

4. Kraus PA, Lipman J: Coronary embolism causing myocardial infarction. Intens Care Med. 1990, 16:215-216. 10.1007/BF01724807

5. Shamsham F, Safi AM, Pomerenko I, Salciccioli L, Feit A, Clark LT, Alam M: Fatal left main coronary artery embolism from aortic valve endocarditis following cardiac catheterization. Catheter Cardio Inte. 2000, 50:74-77. 10.1002/(SICI)1522-726X(200005)50:1<74::AID-CCD16>3.0.CO;2-P

6. Maqsood K, Sarwar N, Eftekhari H, Lotfi A: Septic coronary artery embolism treated with aspiration thrombectomy: case report and review of literature. Tex Heart Inst J. 2014, 41:437-439. 10.14503/THIJ-133386

7. de Walle SV, Dujardin K: A case of coronary embolism in a patient with paroxysmal atrial fibrillation receiving tamoxifen. Int J Cardiol. 2007, 123:66-68. 10.1016/j.ijcard.2006.11.091

8. Mejia VM, Woo YJ, Herrmann HC: Left main coronary embolism. J Invasive Cardiol. 2006, 18:296.

9. Sprinkle JD, Peasley ED: Fatal coronary-artery embolism after pulmonary resection. N Engl J Med. 1963, 268:1444-1446. 10.1056/NEJM196306272682605

10. Nakazone MA, Tavares BG, Machado MN, Maia LN: Acute myocardial infarction due to coronary artery embolism in a patient with mechanical aortic valve prosthesis. Case Rep Med. 2010, 2010:751857. $10.1155 / 2010 / 751857$

11. Shibata T, Kawakami S, Noguchi T, et al.: Prevalence, clinical features, and prognosis of acute myocardial infarction attributable to coronary artery embolism. Circulation. 2015, 132:241-250. 10.1161/CIRCULATIONAHA.114.015134

12. Prizel KR, Hutchins GM, Bulkley BH: Coronary artery embolism and myocardial infarction: a clinicopathologic study of 55 patients. Ann Intern Med. 1978, 88:155-161. 10.7326/0003-4819-88-2-155

13. Kolodgie FD, Virmani R, Finn AV, Romero ME: Embolic myocardial infarction as a consequence of atrial fibrillation: a prevailing disease of the future. Circulation. 2015, 132:223-226. 10.1161/CIRCULATIONAHA.115.017534

14. Waller B, Waller BF, Fry ETA, Hermiller JB, Peters T, Slack JD: Nonatherosclerotic causes of coronary artery narrowing-Part II. Clin Cardiol. 1996, 19:587-591. 10.1002/clc.4960190712

15. Popovic B, Agrinier N, Bouchahda N, et al.: Coronary embolism among ST-segment-elevation myocardial infarction patients: mechanisms and management. Circ Cardiovasc Interv. 2018, 11:005587. 10.1161/CIRCINTERVENTIONS.117.005587

16. Hernández F, Pombo M, Dalmau R, et al.: Acute coronary embolism: angiographic diagnosis and treatment with primary angioplasty. Catheter Cardio Inte. 2002, 55:491-494. 10.1002/ccd.10122

17. Pifarré R, Grieco J, Sullivan HJ, Scanlon PJ, Johnson SA, Gunnar RM: Coronary embolism: surgical management. Ann Thorac Surg. 1980, 30:564-568. 10.1016/S0003-4975(10)61731-6

18. Frikha Z, Zairi I, Kammoun S, et al.: 049: coronary artery embolism. Archives Cardiovasc Dis Suppl. 2013, 5:16. 10.1016/S1878-6480(13)70979-0

19. Belli G, Pezzano A, Biase AMD, et al.: Adjunctive thrombus aspiration and mechanical protection from distal embolization in primary percutaneous intervention for acute myocardial infarction. Catheter Cardio Inte. 2000, 50:362-370. 10.1002/1522-726X(200007)50:3<362::AID-CCD22>3.0.CO;2-H

20. Executive Steering Committee, on behalf of the ROCKET AF Study Investigators: Rivaroxaban-Once daily, oral, direct factor Xa inhibition Compared with vitamin K antagonism for prevention of stroke and Embolism Trial in Atrial Fibrillation: rationale and design of the ROCKET AF study. Am Heart J. 2010, 159:340-347. 10.1016/j.ahj.2009.11.025 